\title{
ROLE OF MEDICAL REHABILITATION TREATMENT IN POST-POLIO SYNDROME - A CASE REPORT
}

\author{
Dogaru Gabriela ${ }^{1,2}$ Stanescu Ioana ${ }^{1,2}$ Pop Ioana ${ }^{2}$ \\ 1. "Iuliu Hatieganu" University of Medicine and Pharmacy Cluj-Napoca \\ 2. Clinical Rehabilitation Hospital Cluj-Napoca
}

\begin{abstract}
The term post-polio syndrome (PPS) was introduced in 1985 by Halstead. It is characterized by sudden or progressive muscle weakness, new muscular atrophy, muscle pain, fatigue, functional impotence, cold intolerance, after a period of at least 15 years from acute polio virus infection, a period of neurological and functional stability, in the absence of other medical explanation [1]. The reported prevalence of PPS is between 15\% and $80 \%$ of all patients with previous polio virus infections [2, 3]. Poliomyelitis continues to be a public health problem, because the consequences of the disease last throughout life. In Europe, there are about 700,000 persons who survived the infection and are still alive. Nonrandomized studies with kinesitherapy programs with a duration between 6 weeks and 7 months, involving isokinetic and isometric endurance muscle training, have demonstrated an increase of muscle strength in the case of patients with mild or moderate muscle weakness, and a reduction of muscle fatigue $[7,8,9]$. The differential diagnosis of PPS can be difficult because of the need to exclude both neurological and non-neurological conditions that aggravate the pre-existing motor deficit. Rehabilitation programs using therapeutic means: kinesitherapy, thermotherapy, hydrothermotherapy, occupational therapy represent the only way to limit functional deficit and to improve pain, playing an important role in the long-term management and care of patients.
\end{abstract}

Key words: rehabilitation program, post-polio syndrome, kinesitherapy

\section{Introduction}

The term post-polio syndrome (PPS) was introduced in 1985 by Halstead. It is characterized by sudden or progressive muscle weakness, new muscular atrophy, muscle pain, fatigue, functional impotence, cold intolerance, after a period of at least 15 years from acute polio virus infection, a period of neurological and functional stability, in the absence of other medical explanation [1]. The reported prevalence of PPS is between $15 \%$ and $80 \%$ of all patients with previous polio virus infections [2, 3]. The few studies available regarding drug treatment have reported no efficiency of the studied drug agents: steroids, pyridostigmine, amantadine in the management of fatigue and muscle strength $[4,5,6]$. Non-randomized studies with kinesitherapy programs with a duration between 6 weeks and 7 months, involving isokinetic and isometric endurance muscle training, have demonstrated an increase of muscle strength in the case of patients with mild or moderate muscle weakness, and a reduction of muscle fatigue $[7,8,9]$. A randomized study reported a significant reduction of pain, depression and fatigue, as well as an improvement of walking following hydrothermotherapy [10]. Poliomyelitis continues to be a public health problem, because the consequences of the disease last 
throughout life. In Europe, there are about 700,000 persons who survived the infection and are still alive. Rehabilitation programs using physical-kinetic therapeutic means represent the only way to limit functional deficit and to improve pain, playing an important role in the long-term management and care of patients. The aim of the study was to clinically and functionally evaluate the efficiency of an individualized rehabilitation program in the case of a patient with PPS.

\section{Material and method}

MV, aged 69, with a history of poliomyelitis since the age of 1 , had walked with a walking frame and subsequently, with crutches with axillary support since the age of 20. In February 2015, he was admitted for progressive muscle weakness, particularly in the lower limbs, fatigue, muscle atrophy, cold intolerance, inability to stand and walk, pain in the knee joints, which was more intense in the right knee, all of which occurred a month before. For approximately 10 years, he had had increased blood pressure values, curently receiving drug therapy and a low sodium diet. The objective examination of the osteoarticular system showed marked dorsolumbar scoliosis, scapula alata, left scapulohumeral (SH) pain, left $\mathrm{SH}$ stiffness (joint testing evidenced $110^{\circ}$ flexion, $30^{\circ}$ extension, $90^{\circ}$ abduction, $50^{\circ}$ internal rotation, $50^{\circ}$ external rotation), reducible flexum of the right knee, bilateral hollow foot, lower limb muscle atrophy, inability to stand and walk. Active segmental movements were diminished in the lower limbs. Muscle testing: 2/5 right lower limb, 3/5 left lower limb, 4/5 bilateral upper limbs. Imperative micturition, dyspnea, diminished osteotendinous reflexes, right knee crepitus were associated. Paraclinical investigations: biological tests were within normal limits, and functional respiratory tests evidenced mild restrictive ventilatory dysfunction. ECG was normal. On EMG, chronic denervation and rare fasciculations were observed. X-rays of the cervico-dorsolumbar spine showed spondylarthrotic changes. Cervical spine magnetic resonance imaging (MRI) evidenced cervical arthrosis. Right knee X-ray revealed moderate gonarthrosis. Right knee ultrasound evidenced a small amount of fluid in the external suprapatellar recess, without changes in tendons or ligaments.

Drug treatment used: NSAIDs, antispastics, vitamins. The patient was clinically and functionally assessed at the beginning of the rehabilitation treatment and after the two weeks of treatment. Pain on the visual analogue scale (VAS), articular mobility using joint evaluation, muscle strength using muscle testing, transfers and walking were monitored. Rehabilitation treatment consisted of sedative paravertebral massage and toning massage of the upper and lower limbs, posturing with the upper limbs in slight abduction, the elbows and knees in exension, in order to prevent tendinous retractions and joint stiffness. Paraffin packing with analgesic and myorelaxant effect was applied to the left shoulder. Passive mobilizations of the lower limbs were performed in order to prevent possible complications of the immobilization syndrome, such as joint stiffness, increased muscle hypotrophy, immobilization osteoporosis, thrombophlebitis, while active mobilizations of the upper limbs were aimed at increasing the joint range of motion. Transfers in bed as well as from bed to wheelchair, postures facilitating respiration, respiratory gymnastics to improve respiratory function, and walking exercises were also carried out. Isotonic and isometric muscle training in order to prevent muscle strength loss was monitored regarding the exercise duration and the rest period. Stretching exercises 
and muscle relaxation exercises were also performed. The ergotherapy program consisted of techniques for the improvement of ADLs. Knee analgesic laser treatment was administered.

\section{Results}

After two weeks of drug and rehabilitation treatment, the intensity of pain in the right shoulder and knee decreased (initial VAS 7 and 8, respectively, post-treatment VAS 5 and 4, respectively). The patient was able to perform transfers to bed and from bed to wheelchair by himself, having a higher degree of independence in the wheelchair. Mobility in the left scapulohumeral joint was significantly improved $\left(150^{\circ}\right.$ flexion, $70^{\circ}$ extension, $120^{\circ}$ abduction, $70^{\circ}$ external rotation, $70^{\circ}$ internal rotation). Muscle testing: 3/5 right lower limb, 3/5 left lower limb, 4/5 bilateral upper limbs. The continuation of kinesitherapy at home was recommended.

\section{Discussions}

The study revealed the benefits of physical-kinetic therapy in maintaining and increasing joint mobility, improving pain, ameliorating muscle strength and respiratory function. The literature data show that the excessive use of muscles and training can aggravate the symptoms of PPS and may cause a decrease in muscle strength [11]. However, there are no prospective studies demonstrating that increased muscle activity or training can reduce muscle strength compared to the absence of training or minimal muscle activity. Patients with regular physical activity had fewer symptoms and a higher functional level compared to physically inactive patients. The majority of the studies based on physical exercise were conducted under monitoring, with submaximal effort, intermittent pauses and rest periods in order to prevent overstress effects $[11,12]$.

The differential diagnosis of PPS can be difficult because of the need to exclude both neurological and nonneurological conditions that cause the aggravation of the pre-existing motor deficit. It is important to establish the cause of the progressive loss of muscle strength in these patients, using imaging methods (cervical spine MRI or CT), electrophysiological methods (EMG, nerve conduction velocities) that are useful to confirm chronic and continuous denervation and to rule out neuropathies, as well as other tests (biochemical tests, muscle biopsy, CSF examination), aimed at excluding other pathologies that might determine or influence the newly developed symptoms.

The most frequent neurological disorders that may induce the progressive aggravation of peripheral motor deficit are cervical myelopathy, syringomyelia, amyotrophic lateral sclerosis, peripheral motor neuropathies and myopathies. Although imaging examinations consisted of cervical spine MRI, in this case, they allowed to rule out compressive cervical arthrotic myelopathy, and the absence of clinical and EMG signs of sensory involvement excluded the diagnosis of radiculo- or neuropathy. Normal muscle enzyme values and EMG examination allowed to rule out muscle disorders.

In addition to neurological disorders, there are many other conditions that may cause muscle weakness or/and fatigue in patients with post-polio sequelae: depression, scapulohumeral periarthritis and rotator cuff syndrome in axillary crutch users (both diseases causing functional impotence due to pain), ventilatory dysfunction induced by the aggravation of scoliosis (which explains fatigue). Also, the overstrain of deficient limbs and joints or the inadequate use of residual muscle groups can aggravate motor and functional deficit.

\section{Conclusions}

Rehabilitation programs using physical-kinetic methods: massage, kinesitherapy, thermotherapy, 
hydrothermotherapy, electrotherapy, occupational therapy are the only way to limit functional deficit and to improve pain, playing an important role in the long-term management and care of patients.

Long-term prognosis is unfavorable; the regular monitoring of patients and their inclusion in complex medical rehabilitation programs are important in order to ensure the autonomy and improve the quality of life of these patients.

However, rehabilitation is a complex process, involving very high costs both at individual, psychoemotional level and at social level.

It is also important for persons who had poliomyelitis to be monitored by a multidisciplinary team, including a medical rehabilitation specialist, a kinesitherapist, a neurologist, an ergotherapist, and an orthotic specialist. The development of centers specialized in the treatment of post-polio syndrome would be useful.

\section{Bibliography}

1. Halstead LS, Rossi CD. New problems în old polio patients: results of a survey of 539 polio survivors. Orthopedics 1985; 8: 845-850.

2. Dalakas MC. The post-polio syndrome as an evolved clinical entity. Definition and clinical description. Annals of the New York Academy of Sciences 1995; 53: 68-80.

3. Farbu E, Rekand T, Gilhus NE. Post polio syndrome and total health status in a prospective hospital study. European Journal of Neurology 2003; 10: 407-413.

4. Dinsmore S, Dambrosia J, Dalakas MC. A double-blind, placebo-controlled trial of high-dose prednisone for the treatment of post-poliomyelitis syndrome.
Annals of the New York Academy of Sciences 1995; 753: 303-313.

5. Trojan DA, Cashman NR. An open trial of pyridostigmine în postpoliomyelitis syndrome. Canadian Journal of Neurological Sciences 1995; 22: 223-227.

6. Stein DP, Dambrosia JM, Dalakas MC. A double-blind, placebo-controlled trial of amantadine for the treatment of fatigue în patients with the post-polio syndrome. Annals of the New York Academy of Sciences 1995; 753: 296-302.

7. Ernstoff B, Wetterqvist $\mathrm{H}$, Kvist $\mathrm{H}$, Grimby G. Endurance training effect on individuals with postpoliomyelitis. Archives of Physical Medicine \& Rehabilitation 1996; 77: 843-848.

8. Spector SA, Gordon PL, Feuerstein IM, Sivakumar K, Hurley BF, Dalakas MC. Strength gains without muscle injury after strength training în patients with postpolio muscular atrophy. Muscle \& Nerve 1996; 19: 1282-1290.

9. Agre JC, Rodriquez AA, Franke TM. Strength, endurance, and work capacity after muscle strengthening exercise in postpolio subjects. Archives of Physical Medicine \& Rehabilitation 1997; 78: 681-686.

10. Strumse YAS, Stanghelle JK, Utne L, Ahlvin P, Svendsby EK. Treatment of patients with postpolio syndrome in a warm climate. Disability \& Rehabilitation 2003; 25: 77-84.

11. Bennett RL, Knowlton GC. Overwork weakness in partially denervated skeletal muscle. Clinical Orthopedics1958; 15: 22-29.

12. Veicsteinas A, Sarchi P, Mattiotti S, Bignotto M, Belleri M. Cardiorespiratory and metabolic adjustments during submaximal and maximal exercise în polio athletes. Medicina Dello Sport 1998; 51: 361-373. 